\title{
Editors' note: 2021 Special Issue on airway management
}

\author{
Stephan K. W. Schwarz, MD, PhD, FRCPC • Philip M. Jones, MD, MSc, FRCPC
}

Received: 13 June 2021/Revised: 13 June 2021/Accepted: 13 June 2021/Published online: 21 June 2021

(C) Canadian Anesthesiologists' Society 2021

There are few topics as central to anesthesiology and critical care medicine as airway management, and a number of recent developments have led to renewed emphasis on this pivotal aspect of our practice. Globally, in the centre of these has of course been the COVID-19 pandemic. Here in Canada, a recent report in the Journal of the findings from an analysis of a decade's worth of closed civil legal airway-related cases from the Canadian Medical Protective Association that involved anesthesiologists has served as a stark reminder of the catastrophic harm that management errors and guideline adherence failures can produce—in healthy elective patients. ${ }^{1}$

This month (September 2021), we are dedicating an entire Special Issue of the Journal to airway management. In the centre of this Special Issue-the Journal's first since April 2018-are, after their last iteration in $2013^{2,3}$ the two-part 2021 updated consensus-based recommendations for management of the difficult airway by the Canadian Airway Focus Group (CAFG). ${ }^{4,5}$ These take into consideration the body of airway management literature that has appeared since the last update as well as the evolution in equipment and practice patterns-including in particular the increasing penetration of videolaryngoscopy.

S. K. W. Schwarz, MD, PhD, FRCPC ( $\square)$

Department of Anesthesiology, Pharmacology \& Therapeutics, The University of British Columbia, and Department of Anesthesia, St. Paul's Hospital/Providence Health Care, 3rd Floor, Providence Bldg., 1081 Burrard Street, Vancouver, BC V6Z 1Y6, Canada

e-mail: sschwarz_cja@cas.ca

P. M. Jones, MD, MSc, FRCPC

Departments of Anesthesia \& Perioperative Medicine and Epidemiology \& Biostatistics, University of Western Ontario, London, ON, Canada
Speaking of the 2013 CAFG difficult airway management recommendations and other major practice guidelines, Howard et al. report eye-opening results from a survey study in Southwestern Ontario on their dissemination and adoption. ${ }^{6}$ In a thought-provoking accompanying editorial, Lemay et al. emphasize the importance of training and visible leadership, and propose the establishment of a Canadian Anesthesiologists' Society Airway Management Section. ${ }^{7}$ In a second editorial, Duggan elaborates on the plethora of clinical practice guidelines and calls for the adoption of a shared algorithmic Advanced Airway Life Support (AALS) approach to airway management emergencies, akin to what we are well used to from Advanced Cardiac Life Support (ACLS) and Advanced Trauma Life Support (ATLS) approaches. ${ }^{8}$

One of the approaches to securing a difficult airway involves fibreoptic flexible bronchoscope-guided intubation through a supraglottic airway device (SGA). It is not every day that a manuscript ends up on an editor's desk that explores such a guideline-based management algorithm component from a preclinical in vitro perspective. Moser et al. from Austria report their findings from a painstaking investigation of 1,040 combinations of endotracheal tubes and SGAs, illustrating the possible problem of dimensional incompatibility and its downstream implications. ${ }^{9}$ In other words: size does indeed matter.

Whereas guidelines also exist on procedural sedation with the goal to improve patient safety, van Schaik et al. report that nearly one third of adult patients from a Dutch cohort of 5,993 undergoing procedural sedation developed significant hypoxemia and stress the need for adequate monitoring, patient selection, and staff training. ${ }^{10}$ 
Going back to the topic of the COVID-19 pandemic, a primary concern among our global community has of course been the risk of SARS-CoV-2 transmission during intubation. Jen et al. report reassuring results from a randomized controlled trial showing that in patients with normal airway predictors, the use of a barrier enclosure did not significantly prolong time to intubation. ${ }^{11}$ Subramaniam et al. summarize findings of a simulation study on the spread of water droplets during oxygen therapy to shed light on the important practical question as to where it might be safe to stand. ${ }^{12}$ Neilipovitz and Ibey describe the retrieval after over 20 years in storage, testing, and repurposing as emergency backup ventilators during the COVID-19 pandemic of gas-operated single-use ventilator devices that originally had been procured in preparation for possible $\mathrm{Y} 2 \mathrm{~K}$ issues that never materialized. ${ }^{13}$ And for a final article from this Special Issue to be highlighted here, Tümer and Ardiçlı share with us the tale of a toddler who was less than delighted with his concerned healthcare providers' idea to advance a COVID19 swab into her nasopharynx for preoperative testing. ${ }^{14}$ The swab stick fractured and, disconcertingly, a $5-\mathrm{cm}$ fragment disappeared without a trace. As is the case for many a good story, there is a happy end, but we will let you read for yourself.

We sincerely hope that you enjoy perusing this Special Issue of the Journal, which comes out as many of you will hopefully be able to recharge and somewhat recuperate during some much-needed summer vacation time. And, importantly, we hope as always that this month's content will help further our shared overall goal: to improve the outcomes of our patients. Our gratitude goes to all our authors, reviewers, and our amazing colleagues on the Editorial Board for making this this Special Issue possible. Stay tuned for more: a number of upcoming Special Issues on topics of broad importance and interest are in the Journal's pipeline. Until then, please stay safe and sound.

\section{Note de la rédaction: Numéro spécial 2021 sur la prise en charge des voies aériennes}

Il y a peu de sujets aussi centraux à l'anesthésiologie et à la médecine des soins intensifs que la prise en charge des voies aériennes, et plusieurs développements récents ont conduit à un regain d'intérêt pour cet aspect crucial de notre pratique. Au coeur de ces développements se trouve évidemment, à l'échelle mondiale, la pandémie de COVID19. Au Canada, un compte rendu récemment publié dans le
Journal rapportait les conclusions de l'analyse d'une décennie de réclamations judiciaires civiles réglées liées aux voies aériennes de l'Association canadienne de protection médicale qui impliquait des anesthésiologistes : cette analyse a servi de rappel brutal des conséquences catastrophiques que les erreurs de prise en charge et le non-respect des lignes directrices peuvent entraîner - chez des patients en bonne santé subissant des chirurgies non urgentes. ${ }^{1}$

Ce mois-ci (septembre 2021), nous consacrons un Numéro spécial entier du Journal au thème de la prise en charge des voies aériennes. Au centre de ce Numéro spécial - le premier depuis avril 2018 - vous trouverez, après leur dernière publication en $2013^{2,3}$ une mise à jour 2021 en deux parties des recommandations consensuelles pour la prise en charge des voies aériennes difficiles par le Canadian Airway Focus Group (CAFG). ${ }^{4,5}$ Ces recommandations prennent en considération l'ensemble de la littérature sur la prise en charge des voies aériennes publiée depuis la dernière mise à jour, ainsi que l'évolution des équipements et des modes de pratique - y compris, en particulier, l'utilisation de plus en plus répandue de la vidéolaryngoscopie.

À ce sujet, Howard et coll. rapportent les résultats révélateurs d'une étude menée dans le sud-ouest de l'Ontario sur la diffusion et l'adoption des recommandations de 2013 du CAFG sur la prise en charge des voies aériennes difficiles et d'autres lignes directrices de pratique importantes. ${ }^{6}$ L'éditorial accompagnant ce compte rendu porte à réfléchir : en effet, Lemay et coll. mettent l'accent sur l'importance de la formation et d'un leadership visible, et proposent la création d'une section dédiée à la prise en charge des voies aériennes au sein de la Société canadienne des anesthésiologistes. ${ }^{7}$ Dans un deuxième éditorial, Duggan approfondit le sujet de la pléthore de lignes directrices de pratique clinique et appelle à l'adoption d'un algorithme partagé pour une approche fondée sur des Soins avancés en réanimation respiratoire (SARR) pour les urgences de prise en charge des voies aériennes, soit une approche similaire à celles que nous connaissons bien pour les Soins avancés en réanimation cardiovasculaire (SARC, ou ACLS en anglais) et les Soins avancés de réanimation des patients polytraumatisés (ATLS - Advanced Trauma Life Support). ${ }^{8}$

L'intubation guidée par bronchoscope fibroscopique flexible via un dispositif supraglottique (DSG) constitue l'une des approches pour prendre en charge des voies aériennes difficiles. Ce n'est pas tous les jours qu'un rédacteur voit sur son bureau un manuscrit qui explore un tel algorithme de prise en charge basée sur des lignes directrices à partir d'une perspective préclinique in vitro. Les Autrichiens Moser et coll. rapportent les résultats de leur évaluation rigoureuse de 1040 combinaisons de tubes 
endotrachéaux et de DSG, illustrant le problème potentiel d'une incompatibilité dimensionnelle et ses implications en aval. ${ }^{9}$ Autrement dit: la taille est effectivement importante.

Alors qu'il existe également des lignes directrices sur la sédation procédurale ayant pour but d'améliorer la sécurité des patients, van Schaik et coll. rapportent que près d'un tiers des patients adultes d'une cohorte néerlandaise de 5993 personnes subissant une sédation procédurale ont souffert d'une hypoxémie significative. Ces auteurs soulignent la nécessité d'un monitorage, d'une sélection des patients et d'une formation du personnel adéquats. ${ }^{10}$

Pour en revenir au sujet de la pandémie de COVID-19, l'une des principales préoccupations de notre communauté mondiale a bien sûr été le risque de transmission du SRASCoV-2 pendant l'intubation. Les résultats de l'étude randomisée contrôlée de Jen et coll. sont rassurants car ils montrent que, chez des patients ayant des prédicteurs de voies aériennes normales, l'utilisation d'une boîte de protection n'a pas prolongé de manière significative le temps d'intubation. ${ }^{11}$ Subramaniam et coll. résument les résultats d'une étude de simulation sur la propagation de gouttelettes d'eau pendant l'oxygénothérapie afin de faire la lumière sur la question pratique importante de savoir où il est possible de se tenir en toute sécurité. ${ }^{12}$ Neilipovitz et Ibey décrivent la récupération, après plus de 20 ans d'entreposage, les tests sur et la réutilisation d'appareils de ventilation à usage unique fonctionnant au gaz en tant que ventilateurs de sauvetage d'urgence pendant la pandémie de COVID-19. À l'origine, ces appareils avaient été achetés en préparation à d'éventuels problèmes de passage à l'an 2000 - lesquels ne se sont jamais concrétisés. ${ }^{13}$ Dans le dernier article de ce Numéro spécial que nous mentionnerons dans cet éditorial, Tümer et Ardıçlı nous partagent l'histoire d'un bambin qui n'était vraiment pas ravi par l'idée de ses fournisseurs de soins de santé inquiets qui voulaient introduire un écouvillon pour la COVID-19 dans son nasopharynx afin de procéder à un test préopératoire. ${ }^{14}$ Le bâton d'écouvillon s'est rompu et, de manière tout à fait déconcertante, un fragment de $5 \mathrm{~cm}$ a disparu, sans laisser de trace. Comme c'est le cas pour beaucoup de belles histoires, celle-ci finit bien, mais nous vous laisserons la lire par vous-même.

Nous espérons sincèrement que vous apprécierez la lecture de ce Numéro spécial du Journal, qui sort alors que beaucoup d'entre vous seront, espérons-le, en mesure de reprendre des forces et de récupérer pendant des vacances d'été bien méritées. Enfin, fait important, nous espérons comme toujours que le contenu de ce mois-ci contribuera à notre objectif global commun : améliorer les devenirs de nos patients. Nous sommes reconnaissants envers tous nos auteurs, réviseurs et incroyables collègues du Comité de rédaction d'avoir rendu possible ce Numéro spécial. Restez à l'affût : plusieurs autres numéros spéciaux sur des sujets d'importance et d'intérêt généraux sont déjà en cours de développement au Journal. D'ici là, prenez soin de vous.

Acknowledgements Dr. Stephan K. W. Schwarz holds the Dr. Jean Hugill Templeton Chair in Anesthesia, supported by the Dr. Jean Templeton Hugill Endowment for Anesthesia Memorial Fund (The University of British Columbia, Vancouver, BC, Canada) and gratefully acknowledges the Department of Anesthesia, St. Paul's Hospital/Providence Health Care (Vancouver, BC, Canada) for ongoing support.

Disclosures Dr. Stephan K. W. Schwarz is the Editor-in-Chief of the Canadian Journal of Anesthesia. Dr. Philip M. Jones is the Deputy Editor-in-Chief of the Canadian Journal of Anesthesia, and a co-author of the CAFG recommendations papers.

Conflict of interest The authors report no competing financial interests relating to this article.

Editorial responsibility This submission was handled by Dr. Sheila Riazi, Associate Editor, Canadian Journal of Anesthesia.

Remerciements Le Dr Stephan K. W. Schwarz est titulaire de la chaire Dre Jean Hugill Templeton en anesthésie, financé par le Fonds commémoratif Dr. Jean Templeton Hugill Endowment for Anesthesia Memorial Fund (Université de la Colombie-Britannique, Vancouver, C.-B., Canada) et remercie le Département d'anesthésie de l'Hôpital St. Paul/Providence Health Care (Vancouver, C.-B., Canada) pour son soutien continu.

Déclaration Le Dr Stephan Schwarz est le rédacteur en chef du Journal canadien d'anesthésie. Le Dr Philip M. Jones est le rédacteur en chef adjoint du Journal canadien d'anesthésie, et l'un des coauteurs des documents de recommandations du CAFG.

Conflits d'intérêt Les auteurs ne font état d'aucun intérêt financier concurrent lié à cet article.

Responsabilité éditoriale Cet article a été traité par Dre Sheila Riazi, rédactrice adjointe, Journal canadien d'anesthésie.

\section{References}

1. Crosby ET, Duggan LV, Finestone PJ, Liu R, De Gorter $R$, Calder LA. Anesthesiology airway-related medicolegal cases from the Canadian Medical Protection Association. Can J Anesth 2021; 68: 183-95.

2. Law JA, Broemling N, Cooper RM, et al. The difficult airway with recommendations for management - Part 1 - Difficult tracheal intubation encountered in an unconscious/induced patients. Can J Anesth 2013; 60: 1089-118.

3. Law JA, Broemling N, Cooper RM, et al. The difficult airway with recommendations for management - Part 2 - The anticipated difficult airway. Can J Anesth 2013; 60: 1119-38.

4. Law JA, Duggan LV, Asselin M, et al. Canadian Airway Focus Group updated consensus-based recommendations for management of the difficult airway: part 1. Difficult airway management encountered in an unconscious patient. Can J Anesth 2021; DOI: https://doi.org/10.1007/s12630-021-02007-0. 
5. Law JA, Duggan LV, Asselin M, et al. Canadian Airway Focus Group updated consensus-based recommendations for management of the difficult airway: part 2. Planning and implementing safe management of the patient with an anticipated difficult airway. Can J Anesth 2021; DOI: https:// doi.org/10.1007/s12630-021-02008-z.

6. Howard M, Noppens R, Gonzalez N, Jones PM, Payne SM. Seven years on from the Canadian Airway Focus Group Difficult Airway Guidelines: an observational survey. Can J Anesth 2021; DOI: https://doi.org/10.1007/s12630-021-02056-5.

7. Lemay $F$, Asselin $M$, Labrecque $P$. Leadership and teaching in airway management. Can J Anesth 2021; DOI: https://doi.org/10. 1007/s12630-021-02057-4.

8. Duggan $L V$. In a forest of airway guidelines, it's time to make a shared path. Can J Anesth 2021; DOI: https://doi.org/10.1007/ s12630-021-02058-3.

9. Moser B, Kemper M, Kleine-Brueggeney M, Gasteiger L, Weiss $M$. Dimensional compatibility and limitations of tracheal intubation through supraglottic airway devices: a mannequinbased in vitro study. Can J Anesth 2021; DOI: https://doi.org/10. 1007/s12630-021-01993-5.

10. van Schaik EP, Blankman P, Van Klei WA, et al. Hypoxemia during procedural sedation in adult patients: a retrospective observational study. Can J Anesth 2021; DOI: https://doi.org/10. 1007/s12630-021-01992-6.

11. Jen TT, Gusti V, Badh C, et al. The impact of a barrier enclosure on time to tracheal intubation: a randomized controlled trial. Can J Anesth 2021; DOI: https://doi.org/10.1007/s12630-021-02024$\mathrm{Z}$.

12. Subramaniam J, Meeks D, Forbes A, Wong DJ, Ward C, McKechnie A. A simulation study investigating the spread of water droplets during oxygen therapy - where is it safe to stand? Can J Anesth 2021; DOI: https://doi.org/10.1007/s12630-02102003-4.

13. Neilipovitz, D, Ibey AA. Use of Vortran Automatic Resuscitator devices as emergency backup ventilators during the COVID-19 pandemic. Can J Anesth 2021; 10.1007/s12630-021-02038-7.

14. Tümer $M$, Ardç̧lı B. A fractured nasopharyngeal swab in the duodenum of a toddler: an unusual complication of preoperative COVID-19 testing. Can J Anesth 2021; DOI: https://doi.org/10. 1007/s12630-021-01995-3.

Publisher's Note Springer Nature remains neutral with regard to jurisdictional claims in published maps and institutional affiliations. 